\title{
All-dielectric metamaterial frequency selective surface
}

\author{
Jun Wang**, Shaobo Qu*, Liyang Li*, Jiafu Wang*, \\ Mingde Feng*, Hua Ma*, Hongliang $\mathrm{Du}^{*}$ and Zhuo $\mathrm{Xu}^{*} \dagger$ \\ *Science College, Air Force Engineering University \\ Xi'an 710051, P. R. China \\ ${ }^{\dagger}$ Electronic Materials Research Laboratory, Xi' an Jiaotong University \\ Xi'an 710049, P. R. China \\ twangjun563@163.com
}

Received 21 July 2017; Revised 26 August 2017; Accepted 28 August 2017; Published 5 October 2017

\begin{abstract}
Frequency selective surface (FSS) has been extensively studied due to its potential applications in radomes, antenna reflectors, high-impedance surfaces and absorbers. Recently, a new principle of designing FSS has been proposed and mainly studied in two levels. In the level of materials, dielectric materials instead of metallic patterns are capable of achieving more functional performance in FSS design. Moreover, FSSs made of dielectric materials can be used in different extreme environments, depending on their electrical, thermal or mechanical properties. In the level of design principle, the theory of metamaterial can be used to design FSS in a convenient and concise way. In this review paper, we provide a brief summary about the recent progress in all-dielectric metamaterial frequency selective surface (ADM-FSS). The basic principle of designing ADM-FSS is summarized. As significant tools, Mie theory and dielectric resonator (DR) theory are given which illustrate clearly how they are used in the FSS design. Then, several design cases including dielectric particle-based ADM-FSS and dielectric network-based ADM-FSS are introduced and reviewed. After a discussion of these two types of ADM-FSSs, we reviewed the existing fabrication techniques that are used in building the experiment samples. Finally, issues and challenges regarding the rapid fabrication techniques and further development aspects are discussed.
\end{abstract}

Keywords: Frequency selective surface; all-dielectric metamaterial; dielectric materials; Mie theory; dielectric resonator theory.

\section{Introduction}

Frequency selective surface (FSS) is a kind of periodic structure that can be used as spatial filters to transmit or reflect electromagnetic (EM) waves with different operating frequencies, polarizations, and incident angles. ${ }^{1,2}$ Due to its filtering property, FSS is widely investigated and applied in microwave and millimeter-wave areas to construct radomes, antenna reflectors, high-impedance surfaces, as well as EM shields, and absorbers. ${ }^{3-6}$ Conventional FSS usually consists of a 2D periodic array of metallic loops, patches, or their complementary structures printed on a substrate. For practical applications with limited space, subwavelength elements instead of much larger traditional elements have been proposed to design miniaturized-element FSS. ${ }^{7-9}$ As it is well known that metamaterials are composed of subwavelength unit cells, it is convenient to design FSS using the concept of metamaterial. ${ }^{10-13}$

As an alternative to metallic FSS, dielectric materials can be used to design and fabricate FSS or filters. Actually, the study of employing dielectrics in filters design may date back to decades ago. Bertoni et al. ${ }^{14,15}$ studied the frequency selective transmission properties of a periodically modulated dielectric layer, which has a lower absorption loss as compared to the metallic screens in millimeter frequencies.
Park et al. ${ }^{16-18}$ introduced dielectric grating waveguide to study filters, which inspire researchers to develop alldielectric filtering via periodic structures in frame of dielectric grating waveguide. Magnusson et al. ${ }^{19-24}$ designed all-dielectric structures or filters based on guided-mode resonance in microwave frequency and optical frequency regions. Recently, Barton et al. designed all-dielectric FSSs based on guided-mode resonance at microwave frequency. ${ }^{25,26}$ Their all-dielectric FSS was successfully tested in the pass band at a high pulsed microwave with a power of $45.26 \mathrm{MW} / \mathrm{m}^{2}$ and without damage. These pioneer works inspired researchers using dielectric materials in the FSS design.

Dielectric material is a large kind of material. Various dielectric materials possess various physical properties, which are capable of achieving specific functions in realizable applications. For example, composite materials always possess high mechanical strength, and they are easy in precision machining. Microwave ceramics with near zero temperature coefficient of the resonant frequency (TCF) are thermo stable, ${ }^{27-29}$ make them suitable in high power and high temperature. Compared with metallic subwavelength unit cells, dielectric materials made unit cells can be used in more different environments. If we combine these various

This is an Open Access article published by World Scientific Publishing Company. It is distributed under the terms of the Creative Commons Attribution 4.0 (CC-BY) License. Further distribution of this work is permitted, provided the original work is properly cited. 
properties in FSS design, all-dielectric FSSs will possess more functional performance, which is capable of developing more engineering applications.

Among the valuable physical properties, relative permittivity of dielectric materials can be used to modulate EM transmission performance in an artificial route, namely, a route of designing all-dielectric metamaterial. Most dielectric materials with high permittivity and low loss are made of microwave ceramics. When using dielectrics to make all-dielectric metamaterials, many kinds of microwave ceramics can be selected to meet the demand of the design. Zhao et al. ${ }^{30}$ used MgO-doped $\mathrm{Ba}_{0.5} \mathrm{Sr}_{0.5} \mathrm{TiO}_{3}\left(\varepsilon_{r}=1600+4.8 i\right)$ as cubic resonators associated with metallic wires to design all-dielectric metamaterial. Lai et al. ${ }^{31}$ used commercially available bulk $\mathrm{ZrO}_{2}$ (purity $=94 \%, \varepsilon_{r}=33$ and loss tangent $=0.002$ ) to create negative refractive identity via single dielectric resonators (DRs). Lepetit et al. ${ }^{32}$ used $\mathrm{Ba}_{0.4} \mathrm{Sr}_{0.6} \mathrm{Ti}_{0.95} \mathrm{Mn}_{0.05} \mathrm{O}_{3} \quad\left(\varepsilon_{r}=715\right.$ with a $5 \times 10^{-3}$ loss tangent) to design and fabricate a dielectric metamaterial. They also exhibited resonant effective permittivity experimentally, which is issued from the second mode of Mie resonances. Besides changing materials, all-dielectric metamaterial unit cells are also proposed in different shapes, such as rods, ${ }^{33-35}$ spheres, ${ }^{36,37}$ cubes, ${ }^{38,39}$ disks, ${ }^{40,41}$ and rings, ${ }^{42}$ etc. These different geometries are employed to generate various resonant modes, making effective permittivity and permeability to be negative simultaneously or respectively.

In the framework of metamaterials, all-dielectric metamaterial FSS (ADM-FSS) based on high-permittivity ceramic resonators was proposed and studied in recent years. ADM-FSS is a kind of all-dielectric FSS where the unit cells are designed using metamaterial-based elements. Li et al. proposed ADM-FSS at microwave frequencies based on high-permittivity ceramic resonators with bandstop or bandpass response. ${ }^{43,44}$ By mechanically tuning the orientation of the ceramic resonators, $\mathrm{Li}$ et al. proposed reconfigurable all-dielectric FSSs with two adjacent stop bands. ${ }^{45}$ They experimentally showed that the FSS can switch between these two bands. Yu et al. demonstrated a dual-band bandpass all-dielectric FSS, and introduced additional cylindrical DRs to broaden the bandwidth and to sharpen the cut-off frequency. ${ }^{46}$ These works show that the bandpass or bandstop responses arise from impedance matching or mismatching, which is caused by the coupling manner of electric and magnetic resonances.

In this review paper, we have a brief summary of the recent works relating to ADM-FSSs. First, we summarized the basic principle of designing ADM-FSS. Then, useful tools of Mie theory and DR theory are given which illustrate clearly how they are used in the FSS design. Several design cases are introduced and reviewed according to these design theories. Herein, ADM-FSSs are divided into two types according to their composite manner, namely dielectric particle-based ADM-FSS and dielectric network-based ADMFSS. After a discussion of these two types of ADM-FSSs, we reviewed the existing fabrication techniques that are used to build the experiment samples. Finally, issues and challenges regarding the rapid fabrication techniques and further development aspects are discussed. This paper shows that dielectric materials, especially microwave ceramics with high permittivities, are priority candidates in the current stage to fabricate ADM-FSS with different transmission characteristics.

\section{Design Theory}

\subsection{The basic principle of designing ADM-FSS}

By inserting high-permittivity sub-wavelength dielectric materials into the low-permittivity matrix, the composite medium can be treated as a metamaterial, which always works in the framework of effective medium theory. ${ }^{47,48}$ Therefore, the macroscopic EM response can be described by effective relative permittivity and permeability, which can be retrieved from $S$ parameters by using the Nicholson-RossWeir method. ${ }^{49-51}$ In this case, dielectric materials are working as sub-wavelength DRs. According to the DR theory, ${ }^{52-54}$ varied resonant modes are inspired at designed frequencies by tuning geometrical shapes, size parameters and relative permittivity.

The method of designing metamaterials can be used to design FSSs, and the schematic diagram is shown in Fig. 1. Usually, all-dielectric FSSs have symmetric structures, which can be seen as a two-port symmetric network. ${ }^{55}$ The transmission properties depend on the normalized characteristic impedance $Z_{n}$, which is calculated with the equation of

$$
Z_{n}= \pm \sqrt{\frac{\left(1+s_{11}\right)^{2}-s_{21}^{2}}{\left(1-s_{11}\right)^{2}-s_{21}^{2}}}
$$

The normalized characteristic impedance $Z_{n}$ is equal to the ratio of characteristic impedance $Z_{c}$ of the FSS to the wave impedance of the free space, namely $Z_{n}=Z_{c} / Z_{0}$. This indicates that the transmission properties of FSSs are mainly determined by the characteristic impedance of the FSS, that is, impedance matching for bandpass response, while impedance mismatching for bandstop response.

If we treat this two-port symmetric network as an effective medium, as shown in Fig. 1(b), $Z_{c}$ is determined by effective permittivity and permeability of the proposed ADM-FSS with an equation of $Z_{c}=\sqrt{\mu_{r} / \varepsilon_{r}}$. These effective medium EM parameters are uniformly distributed in the medium. The values often changed abruptly when resonant modes are emerged. The effective relative permittivity and permeability can be easily calculated using $S$ parameters by the parameter retrieval method. Here, the parameter retrieval method has three meanings. The first is to give effective relative permittivity and permeability quantitatively. The second is to show the resonant type explicitly. The third is to explain the abrupt change of the normalized characteristic impedance. 


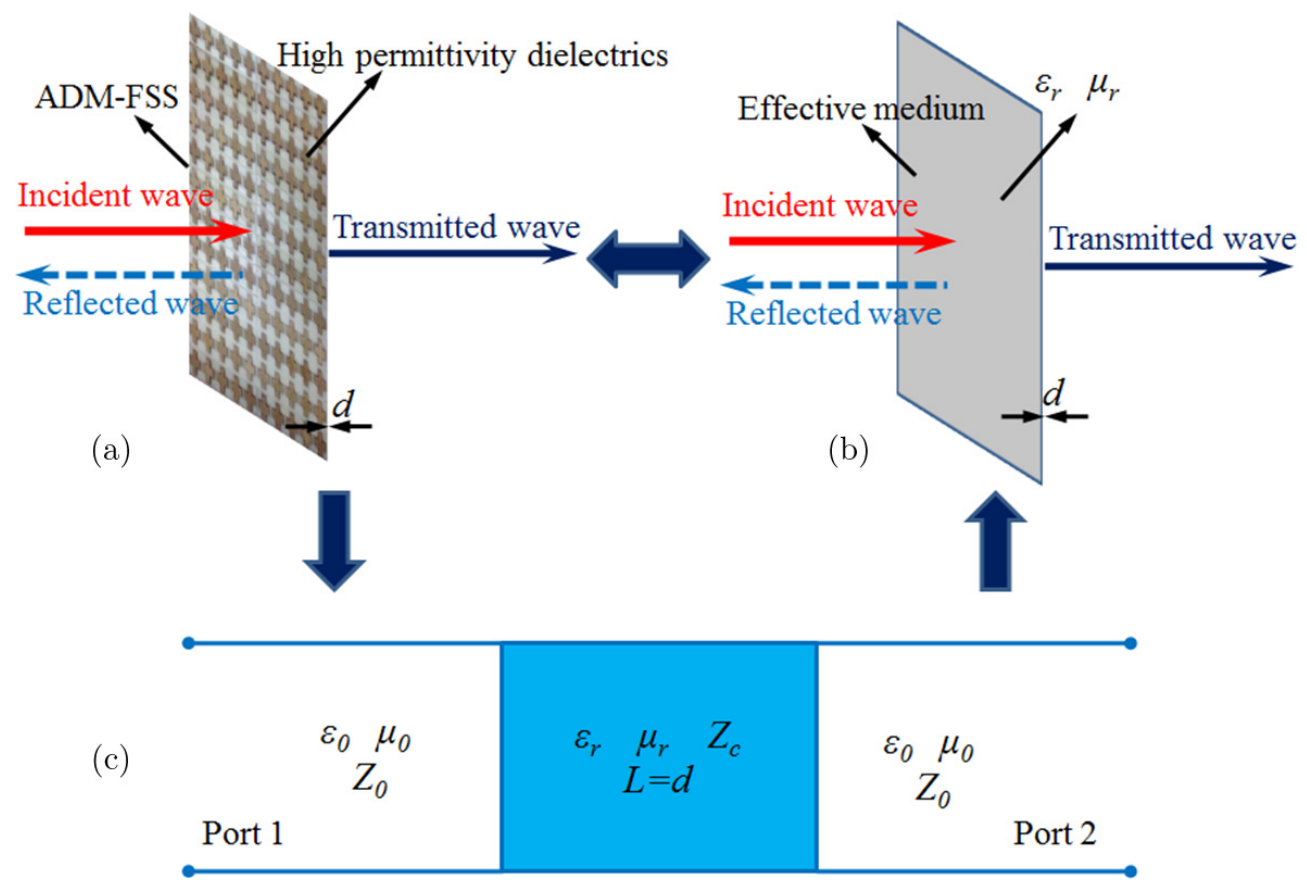

Fig. 1. The basic principle of designing ADM-FSS. (a) Schematic diagram of ADM-FSS, (b) effective medium with abruptly changed permittivity and permeability. (c) two-port symmetric network.

These three meanings make the parameter retrieval method helpful in designing or demonstrating ADM-FSS.

Different DRs can inspire varied resonant modes at designed frequencies. With the ability of tuning resonant modes, the values of effective relative permittivity and permeability can be changed at will. Then, the effective impedance of ADM-FSSs can be designed matching or not in the wanted frequencies. For example, the impedance of metamaterial FSS with single negative permittivity or permeability is difficult to match with free space, and results in a stop band. The impedance of metamaterial FSS with double negative/positive permittivity and permeability may be close to the impedance of free space, leading to a bandpass response.

Thus, the basic principle of designing ADM-FSS is to design the transmission characteristics by tuning the resonant modes of the DRs. That is, to design FSSs with a wanted pass band or stop band, one should first decide the impedance of ADM-FSSs matching or not. Impedance can be tuned at will by changing the frequencies and values of effective EM parameters. Then, according to the DR theory, these effective EM parameters can be changed by tuning geometrical shapes, size parameters and relative permittivities of dielectric inclusions. Finally, in the framework of metamaterial, ADMFSSs can be well designed and fabricated by selecting or combining different resonant modes. This designing method avoids complicated calculations of capacitance and inductance, and provides a new technique route to design FSS.

Two theoretical tools, Mie theory and DR theory, can be used to evaluate the role of the varied resonant modes in tuning bandpass or bandstop responses. These useful tools are introduced as follows.

\subsection{Mie theory}

If the permittivity of dielectric inclusions is much larger than that of the background matrix, the wavelengths inside the dielectric inclusions are comparable to the sizes of the inclusions, while the wavelengths outside the inclusions are larger than the sizes of the inclusions. With this condition, the composite can be described by the effective medium theory. In the framework of effective medium theory, the collection of such inclusions can be treated as an effective material. Thus, the combined scattering response of these collected inclusions can be act as a material with arbitrary values of effective permittivity and permeability. This idea can be verified by using the model proposed in 1947 by Lewin. ${ }^{56} \mathrm{He}$ considered the EM scattering properties of a composite material which are composed of an array of lossless magnetodielectric spheres $\left(\varepsilon_{2}\right.$ and $\left.\mu_{2}\right)$ embedded in a background matrix ( $\varepsilon_{1}$ and $\left.\mu_{1}\right)$. The effective permittivity $\varepsilon_{\text {eff }}$ and permeability $\mu_{\text {eff }}$ expressions based on Mie theory are as follows:

$$
\begin{aligned}
& \varepsilon_{\mathrm{eff}}=\varepsilon_{1}\left(1+\frac{3 \nu}{\frac{\varepsilon_{2} F(\theta)+2 \varepsilon_{1}}{\varepsilon_{2} F(\theta)-\varepsilon_{1}}-\nu}\right), \\
& \mu_{\mathrm{eff}}=\mu_{1}\left(1+\frac{3 \nu}{\frac{\mu_{2} F(\theta)+2 \mu_{1}}{\mu_{2} F(\theta)-\mu_{1}}-\nu}\right) .
\end{aligned}
$$




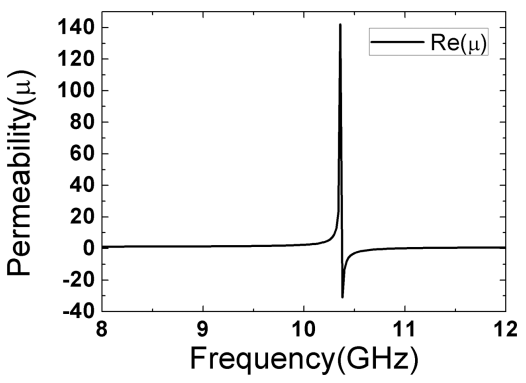

(a)

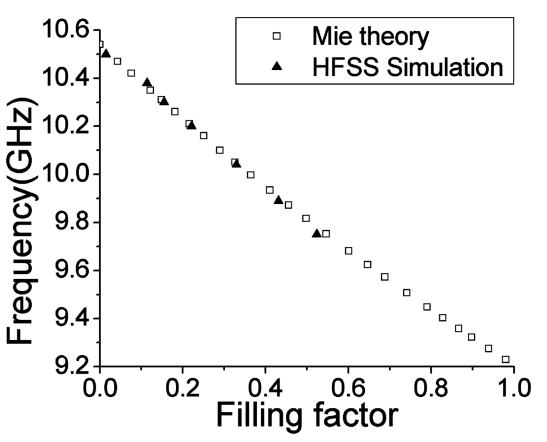

(b)

Fig. 2. (a) The effective permeability according to Lewin's model. (b) Central frequency of the resonant at different filling factors.

where

$$
\begin{gathered}
F(\theta)=\frac{2(\sin \theta-\theta \cos \theta)}{\left(\theta^{2}-1\right) \sin \theta+\theta \cos \theta}, \\
\nu=\frac{4}{3} \pi\left(\frac{r_{0}}{p}\right)^{3} \\
\theta=k_{0} r_{0} \sqrt{\varepsilon_{2} \mu_{2}}
\end{gathered}
$$

$r_{0}$ and $p$ are the inclusion radius and the lattice constant, respectively. The effective EM parameters were formulated only by considering the spheres resonating either in the first or second resonant modes of the Mie series.

If the relative permittivity of the dielectric inclusions is 90 , the inclusion radius $r_{0}$ and the lattice constant $p$ are $1.5 \mathrm{~mm}$ and $5 \mathrm{~mm}$, respectively, as well as let $\varepsilon_{1}$ and $\mu_{1}$ to be 1 . According to Lewin's model, the effective permeability can be calculated as shown in Fig. 1. The abrupt change of the effective permeability is caused by the first resonant modes of the Mie series. The frequency of the maximum effective permeability can be tuned to change the filling factor $\nu$. When $\nu$ approaches 0 , there is only one dielectric sphere in free space. In this case, the magnetic resonant frequency is equal to the first-order TE mode resonant frequency given by Mie scattering theory for a single dielectric sphere. The first-order free resonant frequency is calculated to be $10.54 \mathrm{GHz}$. The central frequency of the resonant can be varied via different filling factors, which provide a technique route to tune the value of the effective permeability.

Mie scattering theory can be used to interpret the origin of negative permittivity and permeability in the framework of effective medium theory. However, it is difficult to demonstrate the detailed resonant modes, especially for dielectric inclusions with complex geometrical shapes. DR theory, with the ability to describe the detailed resonant modes of dielectric inclusions with complex geometrical shapes and complex boundary conditions, is introduced below.

\subsection{Dielectric resonator theory}

Detailed resonant modes can be depicted via DR theory. ${ }^{46-48}$ A dielectric structure with proper size can be regarded as a dielectric cavity at corresponding frequencies. Because the boundary condition of high permittivity dielectrics is a magnetic wall, it would become more perfect for higher permittivity. The incident wave is reflected back and forth inside the dielectric cavity, forming a stationary wave, and behaves as a DR. Usually, the boundaries are not perfect magnetic walls. Thus, DRs do not constraint stationary waves completely. When the localized enhanced EM energy resonates inside the DR, some parts of the energy leak away via the magnetic wall. Then, a $\delta \pi$ phase shift will take place. $\delta$ is increasing from 0 to 1 with the increasing relative permittivity of the DR. This will deduce to a fraction number of stationary waves inside the DR, which means a fraction number of the multiple semi-wavelengths.

There always exist strict solutions for sphere and infinite cylinder DRs to depict the resonant modes. For finite cylinder or rectangular DRs, there exist approximate solutions. For DRs with complex geometrical shapes, there hardly will be strict or approximate solutions. To depict these complicated resonant modes, one can divide the complicated resonant modes into several discrete parts, and seek the similarity of partial modes to standard rectangular DR modes. ${ }^{57}$ Similar resonant modes lead to similar spatial field distributions, and then lead to similar macroscopic EM response. Thus, the resonant modes of a standard rectangular DR are important to depict complicated resonant modes.

The rectangular DR can be calculated with a high accuracy according to the $\mathrm{DR}$ theory. The main resonances of the rectangular DR are $\mathrm{TE}_{(s+\delta), m, n}^{x}$ and $\mathrm{TM}_{(s+\delta), m, n}^{x}$. Considering $\mathrm{TE}_{(s+\delta), m, n}^{x}$ mode, the resonant frequency is calculated via

$$
f_{0}=\frac{c}{2 \pi \sqrt{\varepsilon_{r}}} \sqrt{\left(\frac{(s+\delta) \pi}{a}\right)^{2}+\left(\frac{m \pi}{b}\right)^{2}+\left(\frac{n \pi}{d}\right)^{2}} .
$$

With the equations given in Ref. 58, one can easily calculate the resonant frequency, as well as show detailed resonant modes of any rectangular DR. Varied resonant modes can be inspired by tuning geometrical shapes, size parameters and relative permittivity. Moreover, boundary condition is an important factor that can be employed to modulate the resonant modes. Take rectangular dielectric resonator for 


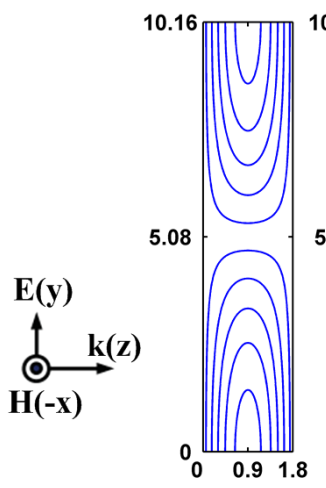

(a)

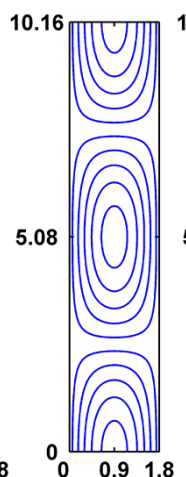

(b)

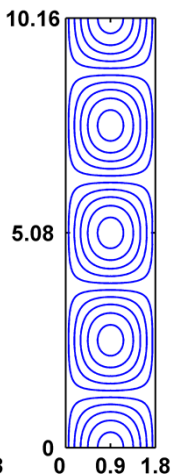

(c)

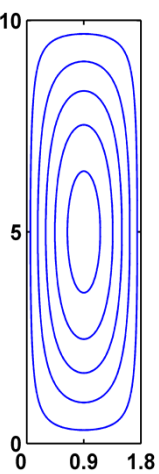

(d)

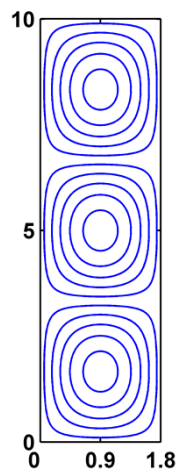

(e)

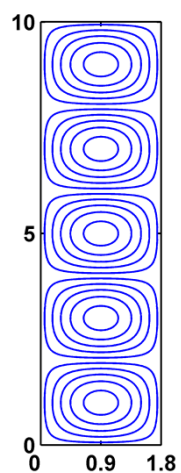

(f)

Fig. 3. Electric field distribution of rectangular-shaped resonator in the $y z$ plane. (a) $\mathrm{TE}_{\delta 11}^{x}$ mode, (b) $\mathrm{TE}_{\delta 21}^{x}$ mode, (c) $\mathrm{TE}_{\delta 41}^{x}$ mode, (d) $\mathrm{TE}_{\delta 11}^{x}$ mode, (e) $\mathrm{TE}_{\delta 31}^{x}$ mode and (f) $\mathrm{TE}_{\delta 51}^{x}$ mode. $^{58}$

example. As shown in Figs. 3(a)-3(c), the two lateral sides in the y-direction can be contacted on the metallic plates. These two sides are perfect $E$-planes, while the rest four lateral sides are $H$-planes due to the high permittivity of the DRs. The electric line of force is perpendicular to the $E$-plane and parallel to the $H$-plane. The modes in Figs. 3(a)-3(c) are $\mathrm{TE}_{\delta 11}^{x}, \mathrm{TE}_{\delta 21}^{x}$, and $\mathrm{TE}_{\delta 41}^{x}$, respectively. As shown in Figs. 3(d)-3(f), all the lateral sides cannot be contacted on metallic plates. These sides should be $H$-planes due to the high permittivity of the DRs. The electric line of force is parallel to the $H$-plane. The modes in Figs. 3(d)-3(f) are $\mathrm{TE}_{\delta 11}^{x}, \mathrm{TE}_{\delta 31}^{x}$, and $\mathrm{TE}_{\delta 51}^{x}$, respectively. Whether there is contact on metallic plate or not, the rectangular DR shows different resonant modes, leading to different EM responses. Thus, boundary conditions can be a useful tool to modulate the transmission characteristic of the ADM-FSS.

\section{Design Cases}

With the design theory in Sec. 2, different types of ADM-FSS were proposed. Some of them are based on discrete dielectric particles, while some are based on continuous dielectric network. In all these design cases, the dielectric materials are made of microwave ceramics. Microwave ceramic is a kind of dielectric materials which can possess high permittivity and low loss. Using different components, microwave ceramic can be designed as thermal sensitive or thermal insensitive. The suitable dielectric materials can be selected by evaluating their mechanical, thermal, and electric properties.

\subsection{Dielectric particle-based ADM-FSS}

Dielectric particles can be designed in different geometrical shapes. Different shapes lead to different resonant modes. Figure 4 shows several types of dielectric particles. Figure 4(a) shows a schematic of ADM-FSS with a stop band in $\mathrm{X}$ band. ${ }^{43}$ Dielectric particles are made of $0.7 \mathrm{Ba}_{0.6} \mathrm{Sr}_{0.4}$ $\mathrm{TiO}_{3}-0.3 \mathrm{La}\left(\mathrm{Mg}_{0.5} \mathrm{Ti}_{0.5}\right) \mathrm{O}_{3}$ ceramic, which has a relative

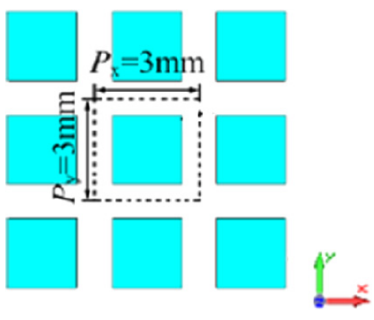

(a)

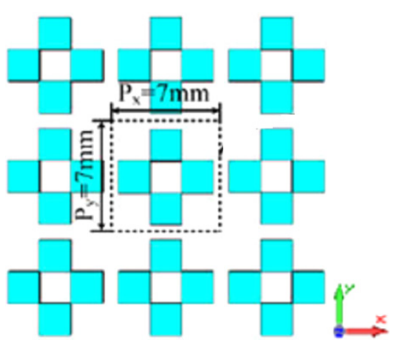

(c)

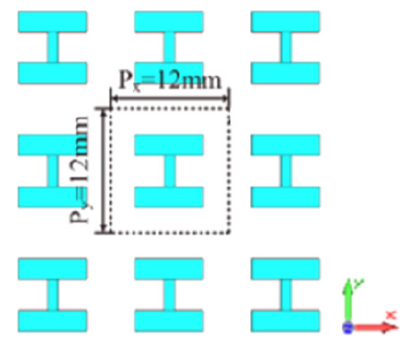

(b)

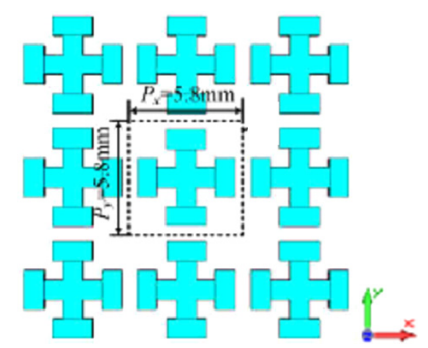

(d)
Fig. 4. Several types of ADM-FSSs with different dielectric particles. (a) A schematic of bandstop ADM-FSS in X band. ${ }^{43}$ (b) Bandpass ADM-FSS. ${ }^{44}$ (c) Bandstop ADM-FSS with building blocks. $^{59}$ (d) Bandstop ADM-FSS with 2D arrays of Jerusalem cross-shaped ceramic resonators. ${ }^{60}$

permittivity of 115 , and a loss tangent of 0.0015 . The ceramic is cut into $2.0 \mathrm{~mm} \times 2.0 \mathrm{~mm} \times 4.0 \mathrm{~mm}$ and placed evenly in a $3 \mathrm{D}$ printed supporting matrix. By adjusting the geometrical parameters of the ceramic resonator, the first two resonant modes are merged to achieve a broadband bandstop property. A nearly $1.5 \mathrm{GHz}$ stop band is demonstrated both numerically and experimentally in the $\mathrm{X}$ band. Figure 4(b) shows a schematic of ADM-FSS with a pass band in the $\mathrm{X}$ band. ${ }^{44}$ Dielectric ceramics were cut into rectangular particles with different sizes. Then, the $H$-shaped structure is assembled by piecing these different rectangular particles together. The new built sub-unit cell can inspire two distinct resonant modes. Each mode causes an impedance mismatch and shows the 


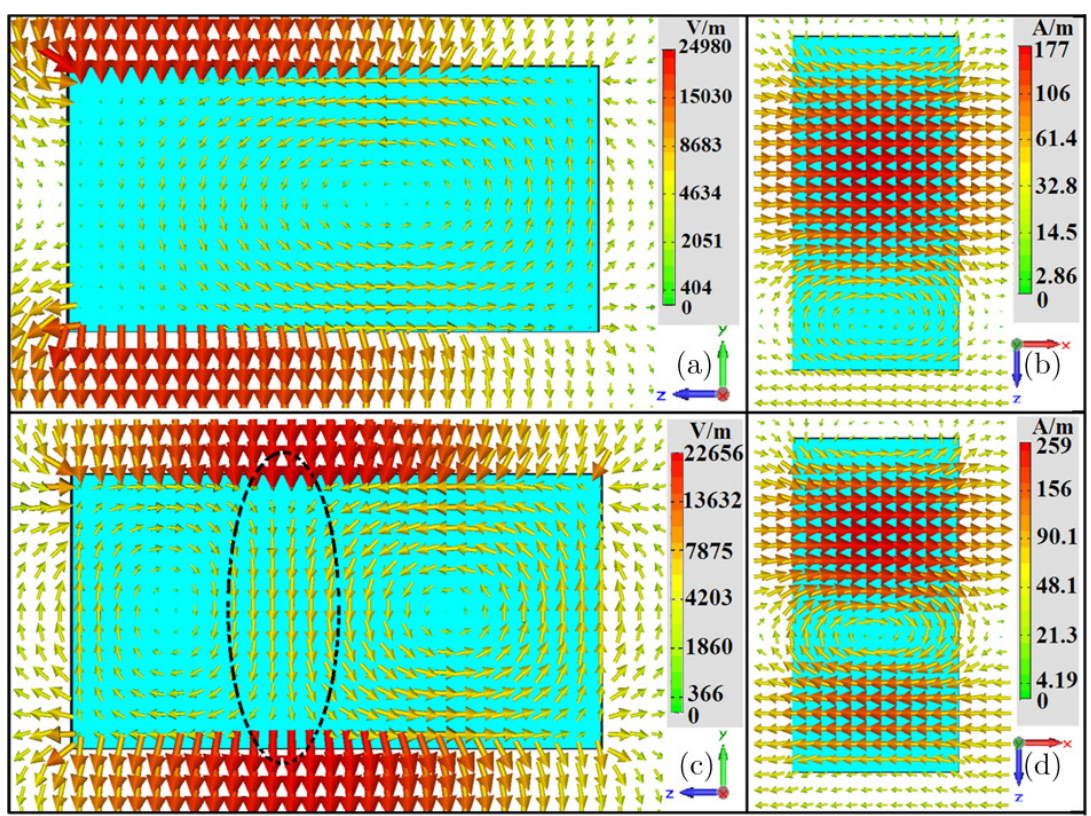

Fig. 5. The distributions of electric fields and magnetic fields. (a) Electric fields at 9.6 GHz. (b) Magnetic field at 9.6 GHz. (c) Electric field at 10.6 GHz. (d) Magnetic field at $10.6 \mathrm{GHz} .^{43}$

prevention of the EM wave. These two modes are far away in the frequency range, and the coupling between them can be neglected. Thus, in the frequency range in between these two modes, the EM wave can pass through the FSS with low insertion loss. With the same idea of building blocks, different unit cells are proposed. ${ }^{59-61}$ The combination of different modes can inspire various resonant behaviors to introduce different impedance characteristics.

The distributions of the electric and magnetic fields corresponding to Fig. 4(a) are shown in Fig. 5. Figures 5(a) and 5(b) show that the electric field formed to a loop in $y z$ plane, which is equivalent to a magnetic dipole at $9.6 \mathrm{GHz}$. Figures 5(c) and 5(d) show that the magnetic field formed to a loop in $x z$ plane, which is equivalent to an electric dipole at 10.6 GHz. The Lorentz-type resonances are inspired inside the DRs, which result in the resonance of the effective permittivity and permeability. Then, the abrupt change of the effective permittivity and permeability makes a band of impedance mismatching in between the two resonant modes.

Figure 6 shows two states of a reconfigurable ADM-FSS which is composed of cross-shaped ceramic resonators. ${ }^{45}$ Based on effective medium theory and DR theory, the

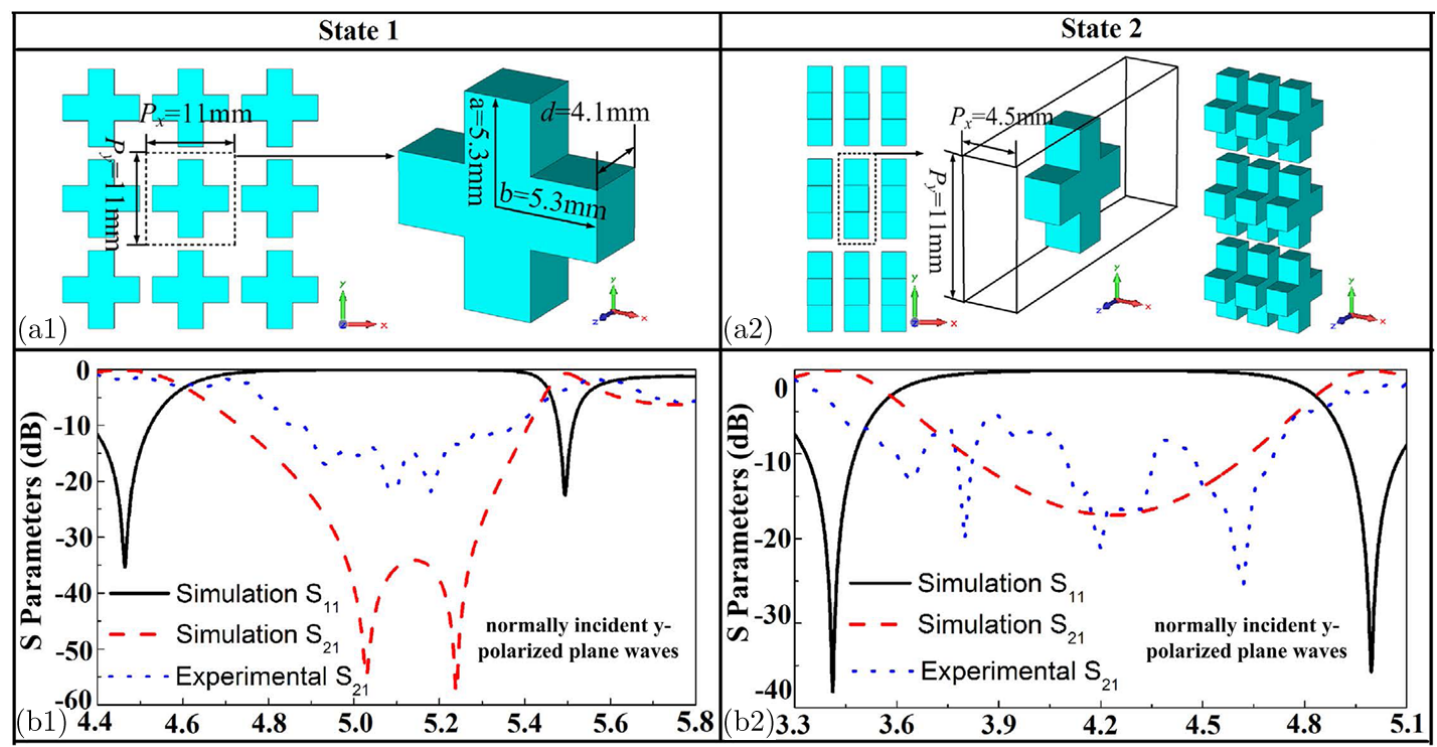

Fig. 6. Unit cell of the reconfigurable ADM-FSS with state 1(a1) and state 2 (a2). Simulation and experimental $S$ parameters of the state 1 (b1) and state 2 (b2) for normally incident $y$-polarized plane waves. ${ }^{45}$ 


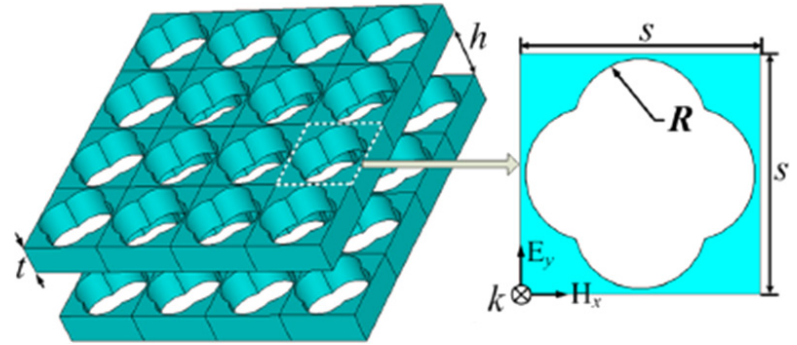

(a)

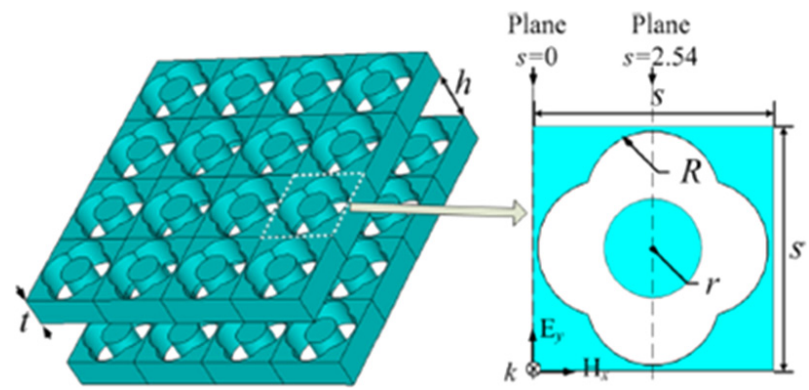

(c)

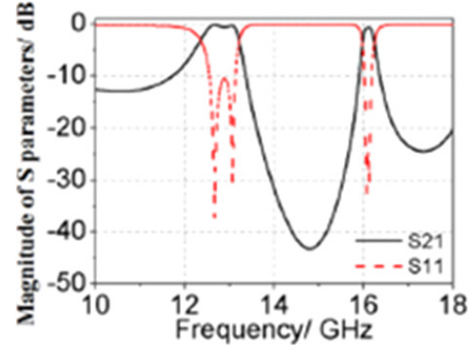

(b)

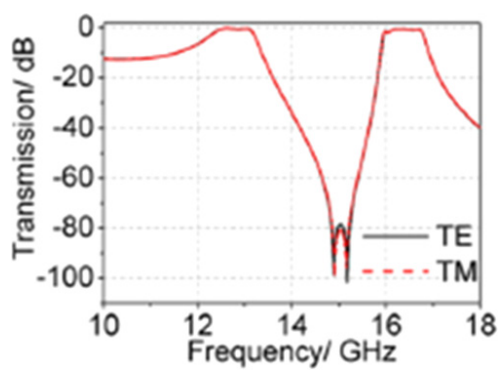

(d)

Fig. 7. Design of dual-band bandpass ADM-FSS in Ku band. (a) The initial FSS composed of CQS cut from dielectric plates. (b) The corresponding bandpass response. (c) The optimized CQS-DR FSS. (d) The corresponding bandpass response with a broader second pass band. ${ }^{46}$

FSS demonstrates different bandstop responses by mechanically tuning the orientation of the ceramic resonators. The two broad stop bands originate from the first two resonant modes of the ceramic resonators. Both numerical and experimental results show that the FSS can switch between two consecutive stop bands in $3.55-4.60 \mathrm{GHz}$ and 4.54-4.94 GHz.

To support the particle-based ADM-FSS, background matrix with low permittivity and low loss tangent should be introduced. Epoxy, light-sensitive silicone, ABS plastics, and aerogel are all possible candidates. Selecting the proper background matrix depends on the needs of the working environment and economical efficiency. Besides the particlebased ADM-FSS, another kind of dielectric network-based ADM-FSS is proposed.

\subsection{Dielectric network based ADM-FSS}

Figure 7(a) shows a dual-band bandpass ADM-FSS in $\mathrm{Ku}$ band, the building elements of which are made of highpermittivity ceramics. ${ }^{46}$ The initial FSS is composed of two-dimensional periodic arrays of complementary quatrefoil structures (CQS) cut from dielectric plates. The structure consists of two dielectric layers separated from each other by air spacing. This double-layer design is always used to enhance the bandpass response. The transmission and reflection coefficients of the FSS under normally incident TE waves are shown in Fig. 7(b). There are two transmission windows in the Ku band. The $-3 \mathrm{~dB}$ bandwidth of the second pass band is $0.3 \mathrm{GHz}(15.948-16.248 \mathrm{GHz})$, and the corresponding fractional bandwidth (FBW) is $1.8 \%$. It is difficult to broaden the bandwidth of the second pass band through this structure only. Nevertheless, DR theory can be used to broaden the bandwidth. By inserting cylindrical dielectric resonators (DRs) in the center of CQS, the optimized CQS-DR FSS is given in Fig. 7(c). The $-3 \mathrm{~dB}$ BW of the second pass band is expanded to $1.28 \mathrm{GHz}(15.92$ $17.11 \mathrm{GHz}$ ), with FBW increased to $7.5 \%$. Further investigations show the that CQS FSS displays a strong electric resonance around $16.8 \mathrm{GHz}$, while the cylindrical DR exhibits a strong magnetic resonance around $16.8 \mathrm{GHz}$. Therefore, a broader pass band can be realized by combining the CQS and cylindrical DR.

The mechanism of the cylindrical DRs broaden the second pass band is illustrated in Fig. 8. By proper designing of the cylindrical DRs, magnetic resonance can be inspired around $16.8 \mathrm{GHz}$. The magnetic resonance can be verified clearly according to the induced field distributions in Figs. 8(d)-8(g). This resonance leads to an impedance mismatching, and results in a transmission dip around $16.8 \mathrm{GHz}$. The impedance mismatching of DRs can be used to improve the impedance of the CQS FSS. This case clearly shows how DR theory works in the ADM-FSS design.

The dielectric network-based ADM-FSS has the merits of polarization independence, stable transmission, and sharp roll-off frequency. The method can also be used to design alldielectric FSSs with continuum structures at other frequencies, as well as to design ADM-FSS with multiple pass bands. ${ }^{62}$ 


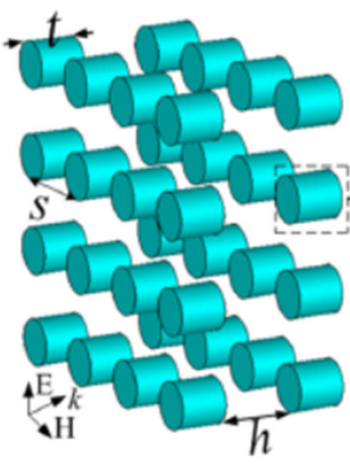

(a)

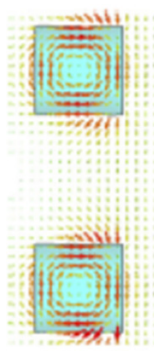

(d)

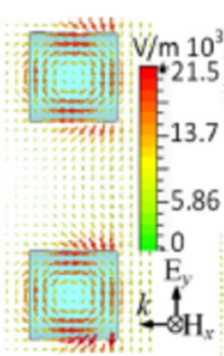

(e)

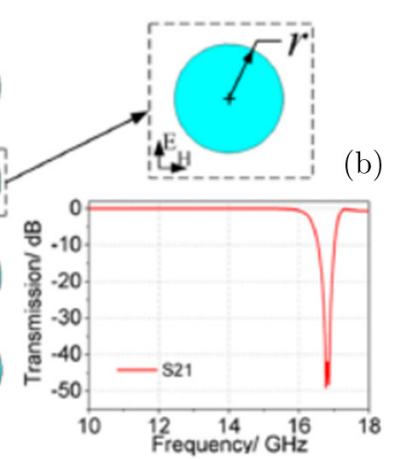

(c)

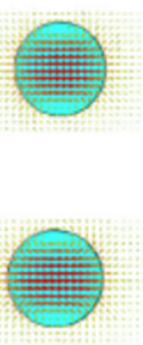

(f)
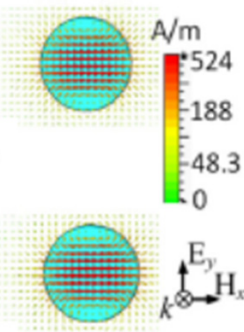

(g)
Fig. 8. (a) Geometry of the cylindrical DR FSS. (b) The unit cell and (c) transmission spectra. Electric field distributions at $16.78 \mathrm{GHz}$ (d) and $16.85 \mathrm{GHz}$ (e). Magnetic field distributions at $16.78 \mathrm{GHz}$ (f) and $16.85 \mathrm{GHz}(\mathrm{g}){ }^{46}$

\section{Fabrication Techniques}

FSS with metallic patches or apertures is always fabricated using the state of art of printed circuit board. It is easy to make metallic FSSs. As an alternative to metallic FSSs, alldielectric FSSs possess the same EM response as the metallic ones, as well as have special advantages. The main issue to prevent realizable applications is the fabrication technique. In the laboratory, several techniques are employed to make small orders. Figure 9 shows three different ADM-FSS samples. They were made with machining and building block method. In this technique, ceramic bulks are made using the solid sintering method ${ }^{63}$ or tape casting method. ${ }^{64}$ Then, the ceramic bulks were cut into small particles with the same or different sizes. Small particles were placed evenly to form an array and then make the ADM-FSS samples, as shown in Figs. 9(a) and 9(c). ${ }^{43,65}$ Moreover, small particles with different sizes are pieced together to make a unit cell with complex geometry. These pieced unit cells are placed in a waveguide to make a simplified testing sample, as shown in Fig. 9(b). Although there is a little difference between the simplified testing sample and the actual FSS sample, the transmission property of the simplified testing sample is consistent with the case testing in free-space. This is due to the reason that the resonant modes which are inspired inside both samples are the same. The building block method provides a simple way to make samples with complex geometrical shapes.

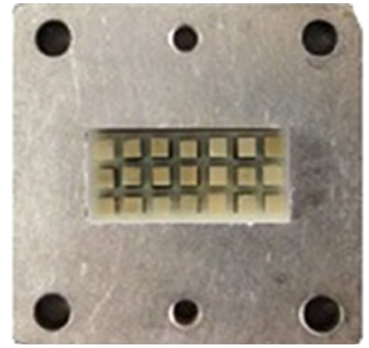

(a)

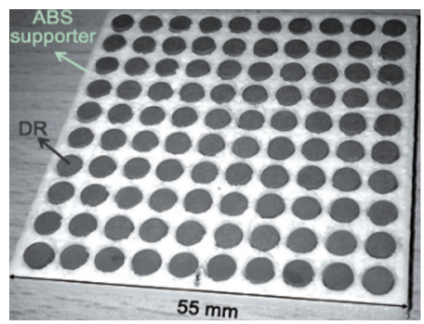

(c)

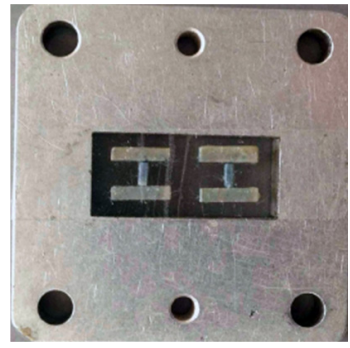

(b)

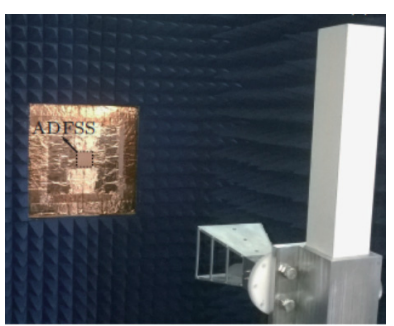

(d)
Fig. 9. (a) ADM-FSS made of cutting machine. ${ }^{43}$ (b) ADM-FSS made of cutting machine and building block method. ${ }^{44}$ (c) ADM-FSS testing sample consists of an array of $(\mathrm{Zr}, \mathrm{Sn}) \mathrm{TiO}_{4} \mathrm{DRs}^{65}$ (d) Experimental setup in the anechoic chamber. ${ }^{65}$

Figure 10 shows another way to make FSS samples with complex geometrical shapes. ${ }^{45}$ The ceramic powders were put into the cross-shaped mould. High pressure was added on the mould. Then the ceramic powders were pressed tightly in the cross shaped mould to form a green-pressing, as shown in Fig. 10(b). Finally, the green-pressing was sintered to ceramics. The cross-shaped ceramics was packaged by a $3 \mathrm{D}$ printed supporting matrix to fabricate the ADM-FSS, as shown in Fig. 10(c). Besides the compression moulding forming technique, the gel-casting method ${ }^{66}$ can also be used to realize near-net shaping of microwave ceramic unit cells.

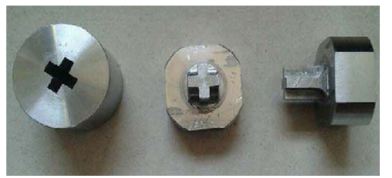

(a)

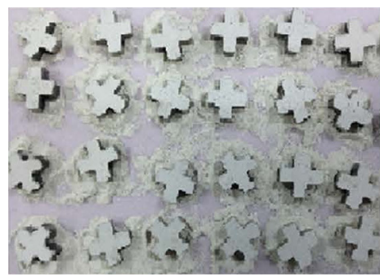

(b)

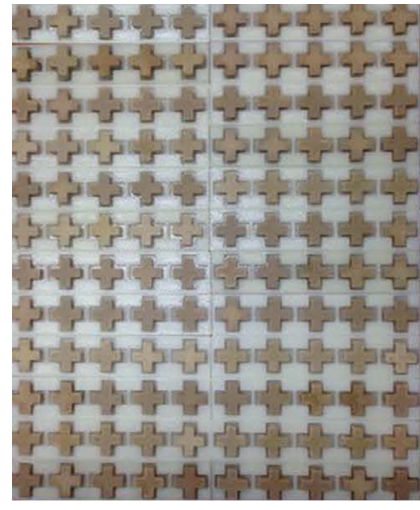

(c)
Fig. 10. (a) The cross shaped steel mould. (b) Ceramic powders made cross shaped green-pressing. (c) ADM-FSS sample composed of cross-shaped ceramics and $3 \mathrm{D}$ printed supporting matrix. ${ }^{45}$ 


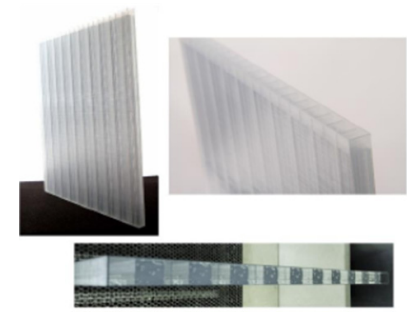

(a)

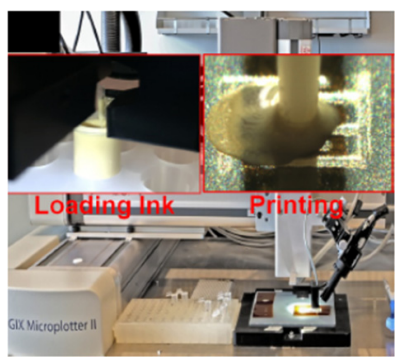

(c)

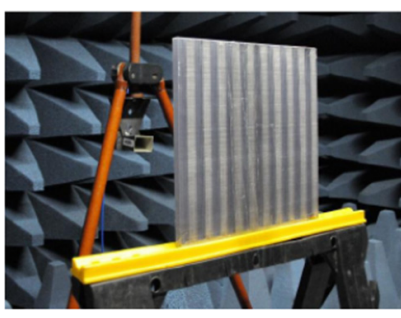

(b)

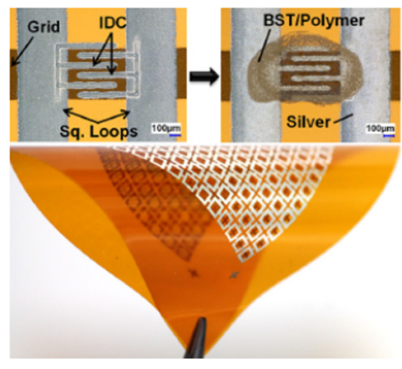

(d)
Fig. 11. (a) Completed 3D printed FSS. ${ }^{26}$ (b) All-dielectric FSS under test with upper C-band horn. ${ }^{26}$ (c) Direct-write printing of reconfigurable FSS. ${ }^{67}$ (d) A printed sample of a flexible FSS. ${ }^{67}$

Actually, there are more fabrication methods that can be introduced to make ADM-FSS. For example, Barton presents a 3D printed all-dielectric FSS and takes a test in the anechoic test facility, as shown in Figs. 11(a) and 11(b). ${ }^{26}$ They used a 3D printer from 3D Systems. The photosensitive polymer resin was used to build a 3D FSS with voids. Once printed, the voids in the FSS were filled with Eccostock HiK 12 powder manufactured by Laird, which has an approximate dielectric constant of 12 and particle sizes of the order of $170 \mu \mathrm{m}$. Haghzadeh et al. demonstrated a fully printed, conformal, bandpass FSS utilizing a novel interdigitated capacitor (IDC) ${ }^{67}$ The 2D FSS consists of an array of metallic square loop and a wire grid printed on either side of a flexible polyimide film using direct-ink writing methodologies. A multiphase barium strontium titanate/cyclic olefin copolymer (BST/COC) composite dielectric ink is printed on the IDCs to vary the resonance frequency of the FSS. These methods can provide rapid and convenient routes of fabricating new types of ADM-FSSs, and enlighten us to develop more manufacturing process to fabricate ADM-FSS in large scales or in short times.

\section{Outlook and Conclusion}

Like conventional metallic FSSs, ADM-FSSs have their potential applications in radomes, antenna reflectors, highimpedance surfaces and absorbers. In the current stage, microwave ceramics with high permittivities are priority candidates to fabricate ADM-FSSs with different transmission characteristics. To fulfill this mission, microwave ceramics should be sintered with a high mechanical strength. Thus, the ceramic fabrication techniques should be improved. To acquire distinct transmission characteristics, shaping techniques such as $3 \mathrm{D}$ printing or direct-write printing should be employed to fabricate ceramic unit cells with complex shapes. These works are significant to promote the development in ADM-FSSs.

Moreover, as emphasized in this review paper, there are abundant physical properties that can be found in different types of dielectric materials. Dielectric materials instead of metallic patterns are capable of achieving more functional performance in the FSS design. By selecting proper dielectric materials, multifunctional ADM-FSSs should be proposed and studied in the near future for more practical applications. For example, ceramics with high permittivity at high temperatures may promote useful applications in extreme environments, including environments of high temperature, high power, and high corrosion. Dielectric materials with excellent mechanical strength and high permittivity can be used to design mechanical metamaterials with a frequency selective property. With this type of dielectric material, it is possible to fabricate devices with a combined structural and functional performance, which is useful to make radomes on missiles or aircrafts.

With the progress in dielectric materials research, ${ }^{68-71}$ other types of dielectric materials may be exploited with a high permittivity at microwaves or even higher frequencies. For example, composite materials, aqueous solutions, and organic solvents are the most possible candidates, due to the fact that they are easier to shape and fabricate. With the ability of rapid fabricating and reconfiguration, dielectric materials composed of aqueous solutions can be used to fabricate ADM-FSS in easy-breaking environments. The more the ability with dielectric materials, the more the functional performance of ADM-FSSs will be. The applications of ADM-FSSs can be expanded with the progress of dielectric materials.

In this paper, we provide a brief summary of the recent progress in ADM-FSS. In the framework of metamaterials, dielectric materials with different high relative permittivities can be used to design ADM-FSS. Several design cases including dielectric particle-based ADM-FSS and dielectric network-based ADM-FSS are introduced and reviewed. After a discussion of these two types of ADM-FSSs, we reviewed the existing fabrication techniques which are used for building the experiment samples. At last, issues and challenges regarding the rapid fabrication and further development aspects are discussed. In conclusion, ADM-FSS has been extensively studied recently. Combined with the advantages of dielectric materials, ADM-FSS demonstrates its great potential in extreme applications. With further development, multifunctional FSS may be put into realization. Dielectric materials have a bright future in the applications of ADM-FSSs. 


\section{Acknowledgments}

The authors are grateful for the support from the National Natural Science Foundation of China under Grant Nos. 11504428, 11274389, 61331005, 61671466, and the National Natural Science Foundation of Shaanxi under Grant Nos. 2016JM6026.

\section{References}

${ }^{1}$ B. A. Munk, Frequency Selective Surfaces: Theory and Design (John Wiley \& Sons, New York, 2000).

${ }^{2}$ B. A. Munk, Finite Antenna Arrays and FSS (John Wiley \& Sons, New York, 2000).

${ }^{3} \mathrm{~N}$. Behdad, A second-order band-pass frequency selective surface using non resonant subwavelength periodic structures, Microw. Opt. Technol. Lett. 50, 1639 (2008).

${ }^{4}$ S. Abadi and N. Behdad, Design of wide band, FSS-based multibeam antennas using the effective medium approach, IEEE Trans. Antennas Progag. 62, 5557 (2014).

${ }^{5}$ S. Abadi, K. Ghaemi and N. Behdad, Ultra-wideband, true-timedelay reflect array antennas using ground-plane-backed, miniaturized element frequency selective surface, IEEE Trans. Antennas Progag. 63, 534 (2016).

${ }^{6}$ G. I. Kiani, A. R. Weily and K. P. Esselle, A novel absorb/transmit FSS for secure indoor wireless networks with reduced multipath fading, IEEE Microw. Wirel. Compon. Lett. 6, 378 (2006).

${ }^{7}$ K. Sarabandi and N. Behdad, A frequency selective surface with miniaturized elements, IEEE Trans. Antennas Progag. 55, 1239 (2007).

${ }^{8}$ C. N. Chiu and K. P. Chang, A novel miniaturized-element frequency selective surface having a stable resonance, IEEE Antennas Wirel. Propag. Lett. 8, 1175 (2009).

${ }^{9}$ G. Yang, T. Zhang, W. Li and Q. Wu, A novel stable miniaturized frequency selective surface, IEEE Antennas Wirel. Propag. Lett. 9, 1018 (2010).

${ }^{10} \mathrm{H}$. Oraizi and M. Afsahi, Design of metamaterial multilayer structures as frequency selective surfaces, Prog. Electromagn. Res. C 6, 115 (2009).

${ }^{11}$ F. Bayatpur and K. Sarabandi, Multipole spatial filters using metamaterial-based miniaturized-element frequency-selective surfaces, IEEE Trans. Microw. Theory Tech. 56, 12 (2008).

${ }^{12}$ F. Bayatpur and K. Sarabandi, A metamaterial-based spatial filter for phased-array applications, IEEE Antennas \& Propagation Society Int. Symp. (2009), pp. 1-4.

${ }^{13}$ B. Sangeetha, G. Gulati, R. U. Nair and S. Narayan, Design of airborne radome using swastika-shaped metamaterial-element based FSS, IEEE India Conference, Bangalore, India, 2017, pp. 1-5.

${ }^{14}$ S. T. Peng, T. Tamir and H. L. Bertoni, Theory of dielectric waveguides, IEEE Trans. Microw. Theory Tech. 23, 123 (1975).

${ }^{15}$ H. L. Bertoni, L. S. Cheo and T. Tamir, Frequency-selective reflection and transmission by a periodic dielectric layer, IEEE Trans. Antennas Propag. 37, 78 (1989).

${ }^{16}$ G. L. Matthaei, D. C. Park, Y. M. Kim and D. L. Johnson, A study of the filter properties of single and parallel-coupled dielectric-waveguide gratings, IEEE Trans. Microw. Theory Tech. 31, 825 (1983).

${ }^{17}$ D. C. Park, G. L. Matthaei and M. S. Wei, Dielectric waveguide grating design for bandstop and bandpass filter applications. IEEE MTT-S Int. Microw. Symp. 84, 202 (1984).
${ }^{18}$ D. C. Park, G. L. Matthaei and M. S. Wei, Bandstop filter design using a dielectric waveguide grating, IEEE Trans. Microw. Theory Tech. 33, 693 (1985).

${ }^{19}$ S. S. Wang, R. Magnusson, J. S. Bagby and M. G. Moharam, Guided-mode resonances in planar dielectric-layer diffraction gratings, J. Opt. Soc. Amer. A. 8, 1470 (1990).

${ }^{20} \mathrm{~S}$. S. Wang and R. Magnusson, Theory and applications of guidedmode resonance filters, Appl. Opt. 32, 2606 (1993).

${ }^{21}$ R. Magnusson, S. S. Wang, T. D. Black and A. Sohn, Resonance properties of dielectric waveguide gratings: Theory and experiments at 4-18 GHz, IEEE Trans. Antennas Propag. 42, 567 (1994).

${ }^{22} \mathrm{R}$. Magnusson and S. S. Wang, Transmission bandpass guidedmode resonance filters, Appl. Opt. 34, 8106 (1995).

${ }^{23}$ Z. S. Liu, S. Tibuleac, D. Shin, P. P. Young and R. Mgnusson, High-efficiency guided-mode resonance filter, Opt. Lett. 23, 1556 (1998).

${ }^{24}$ S. Tibuleac, R. Magnusson, T. A. Maldonado, P. P. Young and T. R. Holzheimer, Dielectric frequency selective structures incorporating waveguide gratings, IEEE Trans. Microw. Theory Tech. 48, 553 (2000).

${ }^{25}$ J. H. Barton, C. R. Garcia, E. A. Berry, R. G. May, D. T. Gray and R. C. Rumpf. All-dielectric frequency selective surface for high power microwaves, IEEE Trans. Antennas Propag. 62, 3652 (2014).

${ }^{26}$ J. H. Barton, C. R. Garcia, E. A. Berry, R. Salas and R. C. Rumpf, 3-D printed all-dielectric frequency selective surface with large bandwidth and field of view, IEEE Trans. Antennas Propag. 63, 1032 (2015).

${ }^{27}$ H. Ogawa, A. Yokoi, R. Umemura and A. Kan, Microwave dielectric properties of $\mathrm{Mg}_{3}\left(\mathrm{VO}_{4}\right)_{2}-x \mathrm{Ba}_{3}\left(\mathrm{VO}_{4}\right)_{2}$ ceramics for LTCC with near zero temperature coefficient of resonant frequency, J. Eur. Ceram. Soc. 27, 3099 (2007).

${ }^{28}$ F. F. Gu, G. H. Chen, X. Q. Li and M. Li, Structural and microwave dielectric properties of the $(1-x) \mathrm{Li}_{3} \mathrm{NbO}_{4}-x \mathrm{Ca}_{0.8}$ $\mathrm{Sr}_{0.2} \mathrm{TiO} 3$ thermally stable ceramics, Mater. Chem. Phys. 167, 354 (2015).

${ }^{29}$ C. Zhang and R. Z. Zuo, Temperature-stable and high Q composite ceramics in low temperature sinterable $\mathrm{BaO}-\mathrm{V}_{2} \mathrm{O}_{5}$ binary system, J. Alloys. Compd. 622, 362 (2015).

${ }^{30}$ Q. Zhao, J. Zhou, F. L. Zhang and D. Lippens, Mie resonancebased dielectric metamaterials, Mater. Today 12, 60 (2009).

${ }^{31}$ Y. J. Lai, C. K. Chen and T. J. Yen, Creating negative refractive identity via single-dielectric resonators, Opt. Express 17, 12960 (2009).

${ }^{32}$ T. Lepetit, E. Akmansoy and J. P. Ganne, Experimental evidence of resonant effective permittivity in a dielectric metamaterial, J. Appl. Phys. 109, 023115 (2011).

${ }^{33}$ L. Peng, L. Ran, H. Chen, H. Zhang, J. A. Kong and T. M. Grzegorczyk, Experimental observation of left-handed behavior in an array of standard dielectric resonators, Phys. Rev. Lett. 98, 157403 (2007).

${ }^{34}$ K. Vynck, D. Felbacq, E. Centeno, A. I. Cabuz, D. Cassagne and B. Guizal, All-dielectric rod-type metamaterials at optical frequencies, Phys. Rev. Lett. 102, 133901 (2009).

${ }^{35}$ L. Peng, L. X. Ran and N. A. Mortensen, Achieving anisotropy in metamaterials made of dielectric cylindrical rods, Appl. Phys. Lett. 96, 241108 (2010).

${ }^{36}$ C. L. Holloway, E. F. Kuester, J. Baker-Jarvis and P. Kabos, A double negative (DNG) composite medium composed of 
magnetodielectric spherical particles embedded in a matrix, IEEE Trans. Antennas Propag. 51, 2596 (2003).

${ }^{37}$ O. G. Vendik and M. S. Gashinova, Artificial double negative media composed by two different dielectric sphere lattices embedded in dielectric matrix, Proc. 34th European Microwave Conf. (IEEE, Amsterdam, 2004), pp. 1209-1212.

${ }^{38}$ Q. Zhao, L. Kang, B. Du, H. Zhao, Q. Xie, X. Huang, B. Li, J. Zhou and L. Li, Experimental demonstration of isotropic negative permeability in a three-dimensional dielectric composite, Phys. Rev. Lett. 101, 027402 (2008).

${ }^{39}$ J. Kim and A. Gopinath, Simulation of a metamaterial containing cubic high dielectric resonators, Phys. Rev. B 76, 115126 (2007).

${ }^{40} \mathrm{O}$. Acher, M. Ledieu, A. Bardaine and F. Levassort, Piezoelectric disks used as metamaterial inclusions, Appl. Phys. Lett. 93, 032501 (2008).

${ }^{41}$ A. Ahmadi and H. Mosallaei, Physical configuration and performance modeling of all-dielectric metamaterials, Phys. Rev. B 77, 045104 (2008).

${ }^{42}$ L. Jelinek and R. Marques, Artificial magnetism and left-handed media from dielectric rings and rods, J. Phys., Condens. Matter 22, 025902 (2010).

${ }^{43}$ L. Y. Li, J. Wang, J. F. Wang, H. L. Du, H. Huang, J. Q. Zhang, S. B. Qu and Z. Xu, All-dielectric metamaterial frequency selective surfaces based on high-permittivity ceramic resonators, Appl. Phys. Lett. 106, 212904 (2015).

${ }^{44}$ L. Y. Li, J. F. Wang, H. Ma, J. Wang, M. D. Feng, H. L. Du, M. B. Yan, J. Q. Zhang, S. B. Qu and Z. Xu, Achieving all-dielectric metamaterial band-pass frequency selective surface via high permittivity ceramics, Appl. Phys. Lett. 108, 122902 (2016).

${ }^{45}$ L. Y. Li, J. Wang, J. F. Wang, H. Ma, H. L. Du, J. Q. Zhang, S. B. $\mathrm{Qu}$ and $\mathrm{Z}$. Xu, Reconfigurable all-dielectric metamaterial frequency selective surface based on high-permittivity ceramics, Sci. Rep. 6, 24178 (2016).

${ }^{46}$ F. Yu, J. Wang, J. F. Wang, H. Ma, H. L. Du, Z. Xu and S. B. Qu, Polarization and angle insensitive dual-band bandpass frequency selective surface using all-dielectric metamaterials, J. Appl. Phys. 119, 134104 (2016).

${ }^{47}$ V. Yannopapas and A. Moroz, Negative refractive index metamaterials from inherently non-magnetic materials for deep infrared to terahertz frequency ranges, J. Phys., Condens. Matter 17, 3717 (2005).

${ }^{48}$ A. Sihvola, Electromagnetic Mixing Formulas and Applications (The Institution of Electrical Engineers, 1999).

${ }^{49}$ A. M. Nicholson and G. F. Ross, Measurement of the intrinsic properties of materials by time-domain techniques, IEEE Trans. Instrum. Measure. 19, 377 (1970).

${ }^{50} \mathrm{~W}$. B. Weir, Automatic measurement of complex dielectric constant and permeability at microwave frequencies, Proc. IEEE, 62, 33 (1974).

${ }^{51}$ D. R. Smith, S. Schultz, P. Markos and C. M. Soukoulis, Determination of effective permittivity and permeability of metamaterials from reflection and transmission coefficients, Phys. Rev. B $\mathbf{6 5}$, 195104 (2002).

${ }^{52}$ D. Kajfez and P. Guillon, Dielectric Resonator, 2nd edn. (Noble Publishing Corporation, Georgia, 1998).

${ }^{53}$ A. Petosa, Dielectric Resonator Antennas Handbook (Artech House, Norwood, MA, 2007).

${ }^{54}$ L. Jylha, I. Kolmakov, S. Maslovski and S. Tretyakov, Modeling of isotropic backward-wave materials composed of resonant spheres, J. Appl. Phys. 99, 043102 (2006).
${ }^{55}$ D. M. Pozar, Microwave Engineering, 3rd edn. (John Wiley \& Sons, NewYork, 2000).

${ }^{56} \mathrm{~L}$. Lewin, The electrical constants of a material loaded with spherical particles, Proc. Inst. Electr. Eng. 94, 65 (1947).

${ }^{57}$ L. Y. Li, J. Wang, H. L. Du, J. F. Wang and S. B. Qu, Achieving a multi-band metamaterial perfect absorbervia a hexagonal ring dielectric resonator, Chin. Phys. B 24, 064201 (2015).

${ }^{58}$ B. Du, J. Wang, Z. Xu, S. Xia, J. F. Wang and S. B. Qu, Band split in multiband all-dielectric left-handed metamaterials, J. Appl. Phys. 115, 234104 (2014).

${ }^{59}$ L. Y. Li, J. Wang, J. F. Wang, H. Ma, M. D. Feng, M. B. Yan, J. Q. Zhang and S. B. Qu, Toward band-stop all-dielectric metamaterial frequency selective surface via dielectric ceramic blocks, IEEE Mtt-s International Microwave Workshop Series on Advanced Materials \& Processes for Rf \& Thz Applications, Chengdu, China, 2016, pp. 1-3.

${ }^{60}$ L. Y. Li, J. Wang, J. F. Wang, H. Ma, M. D. Feng, M. B. Yan, J. Q. Zhang and S. B. Qu, All-dielectric metamaterial band stop frequency selective surface via high-permittivity ceramics, Progress In Electromagnetic Research Symp. (PIERS), Shanghai, China, 8-11 August (2016), pp. 799-803.

${ }^{61}$ L. Y. Li, J. Wang, M. D. Feng, H. Ma, J. F. Wang, H. L. Du and S. B. Qu, All-dielectric metamaterial frequency selective surface based on spatial arrangement ceramic resonators, J. Adv. Dielect. 7, 1750009 (2017).

${ }^{62}$ P. Han, F. Yu, J. Q. Zhang, J. Wang, S. B. Qu, H. Ma, J. F. Wang and Y. Shi, The study of microwave characteristics and design of frequency selective properties based on Barium strontium titanate (in Chinese), Chin. Sci. Bull. 61, 3278 (2016).

${ }^{63}$ J. Wang, Z. Xu, Z. H. Yu, X. Y. Wei, Y. M. Yang, J. F. Wang and S. B. Qu, Experimental realization of all-dielectric composite cubes/rods left-handed metamaterial, J. Appl. Phys. 109, 084918 (2011).

${ }^{64}$ Q. Zhao, B. Du, L. Kang, H. J. Zhao, Q. Xie, B. Li, X. Zhang, J. Zhou, L. T. Li and Y. G. Meng, Tunable negative permeability in an isotropic dielectric composite, Appl. Phys. Lett. 92, 051106 (2008)

${ }^{65}$ Z. B. Wang, C. Gao, B. Li, Z. H. Wu, H. M. Zhang and Y. R. Zhang, All-dielectric frequency selective surface design based on dielectric resonator, Chin. Phys. B 25, 068101 (2016).

${ }^{66}$ W. Liu, Y. X. Wang and Z. X. Xiong, Near-net shaping of microwave ceramics by gel-casting with carrageenan, Key Eng. Mater. 336, 1031 (2007).

${ }^{67}$ M. Haghzadeh and A. Akyurtlu, All-printed, flexible, reconfigurable frequency selective surfaces, J. Appl. Phys. 120, 184901 (2016).

${ }^{68}$ Z. M. Dang, J. K. Yuan, J. W. Zha, P. H. Hu, D. R. Wang and Z. Y. Cheng, High-permittivity polymer nanocomposites: Influence of interface on dielectric properties, J. Adv. Dielect. 3, 1330004 (2013).

${ }^{69}$ L. Zhang and Z. Y. Cheng, Development of polymer-based 0-3 composites with high dielectric constant, J. Adv. Dielect. 1, 1100057 (2011).

${ }^{70}$ D. V. Isakov, Q. Lei, F. Castles, C. J. Stevens, C. R. M. Grovenor and P. S. Grant, 3D printed anisotropic dielectric composite with meta-material features, Mater. Des. 93, 423 (2016).

${ }^{71}$ M. D. Feng, X. Y. Tian, J. F. Wang, M. Yin, S. B. Qu and D. C. Li, Broadband abnormal reflection based on a metal-backed gradient index liquid slab: An alternative to metasurfaces, J. Phys. D, Appl. Phys. 48, 245501 (2015). 